\title{
Propagation dynamics in an autoionization medium
}

\author{
E. Paspalakis, N. J. Kylstra, and P. L. Knight \\ Optics Section, Blackett Laboratory, Imperial College, London SW7 2BZ, United Kingdom
}

(Received 15 December 1998)

\begin{abstract}
We demonstrate that an autoionizing medium can, under specific conditions, become transparent to a short laser pulse propagating in the medium. The interference that leads to transparency is intrinsic to the system and relies on the establishment of a "dark," or "trapped," state that exists as a consequence of Fano interference between direct and resonant photoionization processes. [S1050-2947(99)06607-X]
\end{abstract}

PACS number(s): 42.50.Gy, 32.80.Dz, 42.65.-k, 42.50.Md

\section{INTRODUCTION}

The study of the propagation of electromagnetic pulses in multi-level media has lead to the discovery of a number of interesting phenomena. These include the simultaneous propagation of two pulses having different fundamental frequencies ("simultons") $[1,2]$, electromagnetically induced transparency under matched pulse conditions [3-7] and the creation and propagation of "adiabatons," a general class of pulses which show solitonic behavior in adiabatically evolving atomic systems [8-11]. Pulse propagation effects have also been investigated within other contexts. Examples include the study of lasing without population inversion [12], the influence of the quantized nature of laser fields $[13,14]$, "dragging and cloning" of laser pulses [15], the effects of initial superpositions of the atomic states [16,17], and the effects of Doppler broadening [18]. Furthermore, systems where a spatial excitation can be controlled [19] or even imaged in the temporal profile of laser pulses [20] have been discussed.

In these studies, systems involving bound states were considered. The introduction of dissipative processes are detrimental to, and can even destroy, the coherence required for the manifestation of the above mentioned phenomena. However, recently it has been shown that, using the laser-induced continuum structure (LICS) scheme [21,22], atomic coherence can be preserved even in systems in which dissipative processes (e.g., ionization) are present. These latter studies utilize two lasers in a probe-coupling configuration. Interfering processes arising from the coherent laser-matter interaction involving both of these lasers are necessary for transparency or solitonlike propagation in the medium.

In this paper, we investigate the propagation dynamics of a short laser pulse interacting with an autoionizing medium. We show that Fano interference [23] can lead to transparency in the medium, thereby allowing the laser pulse to propagate without absorption. This phenomenon is closely related to the transparency predicted in a four-level medium via spontaneous-emission interference [24]. The essential feature of the present system is the presence of a continuum of atomic states, so that dissipation is intrinsic to the system. However, the decay processes can interfere and this can give rise to transparency in the medium, as will be discussed below. In contrast to the LICS scheme [21,22], here only a single laser, the probe laser that couples the ground state to both the autoionizing state and the continuum, is involved. The configuration interaction which couples the autoionizing state to the continuum is responsible for the necessary interference in this case, rather than a second laser field.

Since Fano's original work, a number of studies have considered the total absorption of a laser field in the autoionizing medium in the weak-field limit [25]. Numerical studies of the Maxwell-Bloch equations for the pulse propagation have also been carried out [26]. In this article, we re-examine short laser pulse propagation in an autoionizing medium. We place emphasis on establishing the connection between adiabatic population trapping in short, pulsed laser fields [27,28] and transparency in the propagation of the laser pulse in the medium. Furthermore, we calculate both analytically and numerically corrections to the adiabatic behavior and show that in the first approximation the laser pulse retains its shape and only its group velocity is modified.

In the following section we derive the MaxwellSchrödinger equations of motion for the atomic system and the laser field. We then discuss the conditions under which a "dark" (or "trapped") state [29] occurs in the atomic system, and consider the possibility of satisfying these conditions for not too intense laser pulses. In Sec. III we establish the link between adiabatic population trapping in the atomic system and the transparency of the medium to the probe laser pulse. We demonstrate, both analytically and numerically, that when the system evolves adiabatically the propagation of the pulse is both loss- and dispersion-free when the trapping conditions are satisfied. Nonadiabatic effects, which can lead either to the reduction of the group velocity of the pulse or even to both absorption and dispersion, are also discussed. Finally, we summarize and conclude in Sec. IV.

\section{BASIC EQUATIONS}

The system under consideration is shown in Fig. 1, where we depict a laser-driven autoionizing system [27,30,31] which consists of a bound state $|0\rangle$, an autoionizing state $|1\rangle$ and a continuum of states $|c, \epsilon\rangle$. The state $|0\rangle$ is coupled to $|1\rangle$ by a laser field. Both of these states are also coupled to the continuum: the autoionizing state via the configuration interaction $V^{c}$ and the bound state via the laser field. Spontaneous emission and other types of decoherence processes (e.g., collisions) are assumed to be unimportant on the time scales of interest, and we therefore analyze the behavior of the system using the Maxwell-Schrödinger equations. The position and time-dependent Hamiltonian of the atomic system is written as 


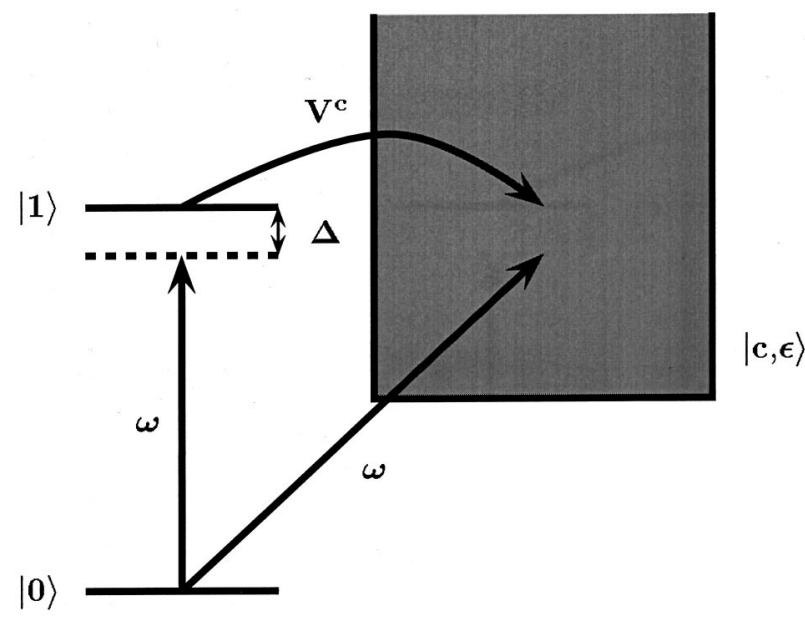

FIG. 1. Schematic diagram of the system under consideration. The initially populated bound state $|0\rangle$ is coupled to the autoionizing state $|1\rangle$ and the continuum $|c, \epsilon\rangle$ by a laser field of angular frequency $\omega$. The autoionizing state $|1\rangle$ is coupled to the continuum by the configuration interaction $V^{c}$.

$$
\begin{aligned}
H= & \sum_{m=0,1} E_{m}|m\rangle\left\langle m\left|+\int_{0}^{\infty} d \epsilon \epsilon\right| c, \epsilon\right\rangle\langle c, \epsilon| \\
& +\left[\int_{0}^{\infty} d \epsilon V_{1 \epsilon}^{c}|1\rangle\left\langle c, \epsilon\left|-\mu_{01} E(z, t)\right| 0\right\rangle\langle 1|\right. \\
& \left.-\int_{0}^{\infty} d \epsilon \mu_{0 \epsilon} E(z, t)|0\rangle\langle c, \epsilon|+\text { H.c. }\right] .
\end{aligned}
$$

Here $E_{m}$ is the unperturbed energy of level $|m\rangle$ (m $=0,1), \quad \epsilon$ is the energy of the continuum state $|c, \epsilon\rangle$ and $V_{1 \epsilon}^{c}=\left\langle 1\left|V^{c}\right| c, \epsilon\right\rangle$. The laser field is described classically as a time- and spatially dependent electric field,

$$
\vec{E}(z, t)=\hat{\varepsilon} E(z, t)=\hat{\varepsilon}[\mathcal{E} f(z, t) \exp i(\omega t-k z)+\text { c.c. }],
$$

where $\omega$ is the angular frequency, $k$ is the wave number, $\hat{\varepsilon}$ is the polarization vector, $\mathcal{E}$ is the electric field amplitude and $f(z, t)$ is the dimensionless pulse envelope of the laser field. The (real) dipole matrix elements coupling the state $|0\rangle$ to, respectively, the autoionizing level $|1\rangle$ and the continuum state $|c, \epsilon\rangle$ are $\mu_{01}=\langle 0|\vec{\mu} \cdot \hat{\varepsilon}| 1\rangle$ and $\mu_{0 \epsilon}=\langle 0|\vec{\mu} \cdot \hat{\varepsilon}| c, \epsilon\rangle$, with $\vec{\mu}$ being the dipole moment operator.

The wave function of the system is first expanded in terms of the bare atomic states multiplied by the corresponding space- and time-dependent amplitudes $a_{m}(z, t) \quad(m=0,1)$ and $a_{\epsilon}(z, t)$ of, respectively, the bound and continuum states (with $\hbar=1$ )

$$
\begin{aligned}
|\psi(z, t)\rangle= & \sum_{m=0,1} a_{m}(z, t) \mathrm{e}^{-i E_{m} t}|m\rangle \\
& +\int_{0}^{\infty} d \epsilon a_{\epsilon}(z, t) \mathrm{e}^{-i \epsilon t}|c, \epsilon\rangle .
\end{aligned}
$$

We substitute this expansion into the time-dependent Schrödinger equation

$$
i \frac{\partial}{\partial t}|\psi(z, t)\rangle=H|\psi(z, t)\rangle,
$$

and obtain the formal solution of $a_{\epsilon}(z, t)$ after the rotating wave approximation is made,

$$
\begin{aligned}
a_{\epsilon}(z, t)= & i\left[\int_{0}^{t} d t^{\prime} \mu_{\epsilon 0} \mathcal{E} f^{*}\left(z, t^{\prime}\right) a_{0}\left(z, t^{\prime}\right) \mathrm{e}^{i\left[\left(\epsilon-E_{0}-\omega\right) t^{\prime}+k z\right]}\right. \\
& \left.-V_{\epsilon 1}^{c} \int_{0}^{t} d t^{\prime} a_{1}\left(z, t^{\prime}\right) \mathrm{e}^{i\left(\epsilon-E_{1}\right) t^{\prime}}\right]
\end{aligned}
$$

Eliminating $a_{\epsilon}(z, t)$ and performing the change of variables,

$$
\begin{gathered}
c_{0}(z, t)=a_{0}(z, t), \\
c_{1}(z, t)=a_{1}(z, t) e^{-i\left(E_{1}-E_{0}-\omega\right) t-i k z},
\end{gathered}
$$

results in the following set of equations in the rotating wave and the Markov approximations [27]:

$$
i \frac{\partial}{\partial t} \mathbf{c}(z, t)=\mathbf{H}(z, t) \mathbf{c}(z, t) \text {. }
$$

The effective, non-Hermitian Hamiltonian is

$$
\mathbf{H}(z, t)=\left(\begin{array}{cc}
\left(\delta E_{0}-\frac{i}{2} \Gamma_{0}\right)|f(z, t)|^{2} & -\frac{1}{2}(q+i) \sqrt{\Gamma_{0} \Gamma_{1}} f(z, t) \\
-\frac{1}{2}(q+i) \sqrt{\Gamma_{0} \Gamma_{1}} f^{*}(z, t) & \Delta-\frac{i}{2} \Gamma_{1}
\end{array}\right),
$$

and $\mathbf{c}(z, t)=\left[c_{0}(z, t), c_{1}(z, t)\right]^{T}$. The intensity-dependent ac Stark shift and photoionization rate of level $|0\rangle$ are given by, respectively, $\delta E_{0}|f(z, t)|^{2}$ and $\Gamma_{0}|f(z, t)|^{2}$, where $\Gamma_{0}$ $=2 \pi\left|\mu_{0 \epsilon} \mathcal{E}\right|^{2}$ and their values are taken at resonance. The laser detuning from resonance is $\Delta=E_{1}+\delta E_{1}-E_{0}-\omega$ and the Fano asymmetry parameter [23] is denoted by $q$ and is defined as

$$
q=\frac{1}{2 \pi \mu_{0 \epsilon} V_{\epsilon 1}^{c}}\left[\mu_{01}-\mathcal{P} \int_{0}^{\infty} d \epsilon^{\prime} \frac{\mu_{0 \epsilon^{\prime}} V_{\epsilon^{\prime} 1}^{c}}{\left(\epsilon^{\prime}-\epsilon\right)}\right]
$$

The autoionization rate $\Gamma_{1}=2 \pi\left|V_{1 \epsilon}^{c}\right|^{2}$ and shift $\delta E_{1}$ are due to the coupling of state $|1\rangle$ to the continuum. The parameters $\Gamma_{1}, \delta E_{1}$, and $q$ are taken to be constants and are evaluated at 
resonance. The term $\sqrt{\Gamma_{0} \Gamma_{1}} f(z, t)(i+q) / 2$ is responsible for the Fano interference intrinsic to this system, as the intermediate state $|1\rangle$ decays to the electronic continuum via two different, but indistinguishable, paths.

To complete the set of required equations for the study of the propagation of a laser pulse in this medium, we also need the Maxwell wave equation describing the propagation of the laser pulse. Within the slowly varying envelope approximation $[32,33]$ this equation is

$$
\left[\frac{\partial}{\partial z} f(z, t)+\frac{1}{c} \frac{\partial}{\partial t} f(z, t)\right] \mathcal{E} e^{i(\omega t-k z)}=-\frac{2 i \pi \omega}{c} P(z, t) .
$$

The (negative frequency part of the) macroscopic polarization of the medium is given by, assuming only homogeneous broadening,

$$
\begin{aligned}
P(z, t)= & \mathcal{N}\left[\mu_{10} a_{0}(z, t) a_{1}^{*}(z, t) e^{i\left(E_{1}-E_{0}\right) t}\right. \\
& \left.+\int_{0}^{\infty} d \epsilon \mu_{\epsilon 0} a_{0}(z, t) a_{\epsilon}^{*}(z, t) e^{i\left(\epsilon-E_{0}\right) t}\right] .
\end{aligned}
$$

Substituting Eq. (5) into Eq. (11) and, after making the Markov approximation, we obtain the following equation for the laser field envelope:

$$
\begin{aligned}
{\left[\frac{\partial}{\partial z}+\frac{1}{c} \frac{\partial}{\partial t}\right] f(z, t)=} & i \alpha\left[\left(\delta E_{0}+\frac{i}{2} \Gamma_{0}\right) f(z, t)\left|c_{0}(z, t)\right|^{2}\right. \\
& \left.+\frac{1}{2} \sqrt{\Gamma_{0} \Gamma_{1}}(i-q) c_{0}(z, t) c_{1}^{*}(z, t)\right] .
\end{aligned}
$$

Here, $\alpha=2 \pi \mathcal{N} \omega / c \mathcal{E}^{2}$ is proportional to the propagation constant and has the dimensions of inverse velocity, and $\mathcal{N}$ denotes the density of atoms in the medium. Equations (8) and (12) are then transformed to the retarded (local) frame where $\tau=t-z / c$ and $\zeta=z$, which is convenient for numerical calculations [32,33]. In this frame, $\partial / \partial \tau=\partial / \partial t$ and $\partial / \partial \zeta$ $=\partial / \partial z+c^{-1} \partial / \partial t$; hence, Eqs. (7) and (12) can be cast in the form

$$
\begin{gathered}
i \frac{\partial}{\partial \tau} \mathbf{c}(\zeta, \tau)=\mathbf{H}(\zeta, \tau) \mathbf{c}(\zeta, \tau), \\
\frac{\partial}{\partial \zeta} f(\zeta, \tau)=i \alpha\left[\left(\delta E_{0}+\frac{i}{2} \Gamma_{0}\right) f(\zeta, \tau)\left|c_{0}(\zeta, \tau)\right|^{2}\right. \\
\left.+\frac{1}{2} \sqrt{\Gamma_{0} \Gamma_{1}}(i-q) c_{0}(\zeta, \tau) c_{1}^{*}(\zeta, \tau)\right]
\end{gathered}
$$

We now discuss the condition under which a dark (or trapped) state [29] can appear in this system. It has been shown in other systems that dark states can be important in obtaining transparency in atomic media $[3,24,34]$. The population trapping condition for our system has been recently discussed by Paspalakis et al. [27]. It is obtained by searching for a real solution of the characteristic equation of the non-Hermitian Hamiltonian $\mathbf{H}$,

$$
\begin{gathered}
\left(\delta E_{0}|f(\zeta, \tau)|^{2}-\frac{i}{2} \Gamma_{0}|f(\zeta, \tau)|^{2}-E\right)\left(\Delta-\frac{i}{2} \Gamma_{1}-E\right) \\
-\frac{1}{4}(q+i)^{2} \Gamma_{0} \Gamma_{1}|f(\zeta, \tau)|^{2}=0 .
\end{gathered}
$$

By setting the imaginary part of Eq. (15) to zero and solving for $E$ we obtain that, for time- and space-dependent laser pulses, the laser detuning must satisfy the condition

$$
\Delta=-\frac{q}{2} \Gamma_{1}+\left(\frac{q}{2} \Gamma_{0}+\delta E_{0}\right)|f(\zeta, \tau)|^{2}
$$

for a dark state to occur in the system. Obviously, this requirement is not satisfied in general, since the second term on the right-hand side (rhs) is space and time dependent. However, if

$$
\frac{q}{2} \Gamma_{0}=-\delta E_{0},
$$

then the rhs of Eq. (16) becomes time and space independent. In this case, a dark state exists if the laser is tuned such that

$$
\Delta=-\frac{q}{2} \Gamma_{1} .
$$

For a not too intense laser field, i.e., $\Gamma_{0} \ll \Gamma_{1}$, the fulfilment of condition (17) is not as important as the fulfilment of condition (18) for the creation of a dark state. In this case, the problematic second (space- and time-dependent) part of the rhs of Eq. (16) is only a small correction to the trapping condition and thus, as a first approximation, its contribution can be omitted. Indeed, typically $\Gamma_{1} \sim 10^{-3}$ a.u., so that for a laser intensity of $10^{10} \mathrm{~W} \mathrm{~cm}^{2}$, the ratio $\Gamma_{0} / \Gamma_{1}$ would be in the range of $10^{-2}$ to $10^{-4}$, depending on the atomic system considered. Also, typically $\delta E_{0} / \Gamma_{0} \sim 1$ [35]. This issue will be addressed in the numerical calculations below. We note that the effective two-level model utilized here has been shown to remain applicable even at intensities at which $\Gamma_{0}$ $\sim \Gamma_{1}[36]$.

\section{PROPAGATION DYNAMICS}

Let us now make the connection between adiabatic coherent population trapping and loss-free pulse propagation in this medium. When Eq. (16) is satisfied, the "dark" state of the system is given by

$$
\mathbf{c}_{\text {dark }}(\zeta, \tau)=N(\zeta, \tau)\left(\begin{array}{c}
\sqrt{\Gamma_{1}} \\
-\sqrt{\Gamma_{0}} f^{*}(\zeta, \tau)
\end{array}\right),
$$

with $N(\zeta, \tau)$ being a normalization factor. This "dark" eigenstate is adiabatically connected to the ground state of the system [27,28]. Therefore, if the laser pulse is applied adiabatically and the laser and atomic parameters are such that Eq. (16) is fulfilled, the system will adiabatically evolve into the dark state. When this is the case, we have 


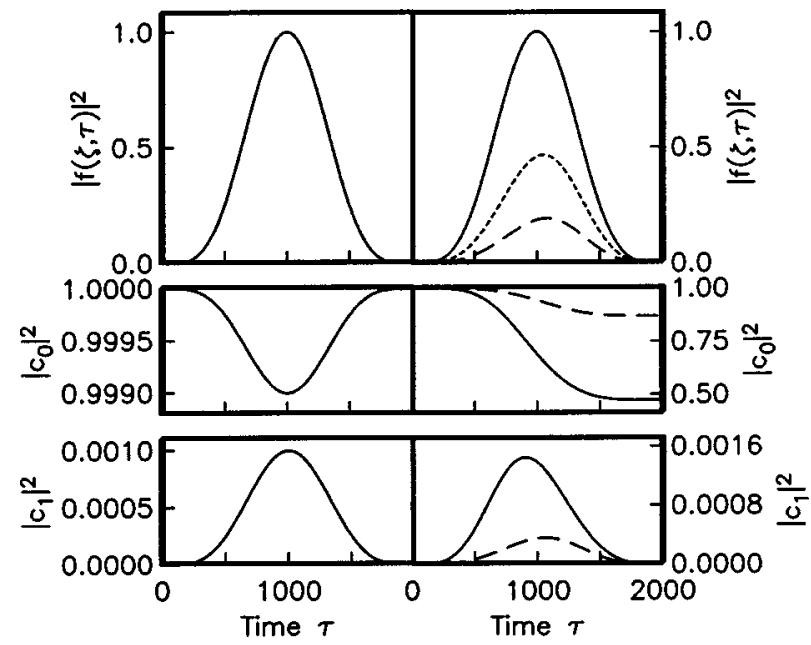

FIG. 2. Magnitude squared of the pulse envelope and the atomic populations as a function of $\tau$ for different values of $\zeta$, with $\alpha \zeta$ $=0$ (solid curves), $\alpha \zeta=\alpha \zeta_{\max } / 2$ (dotted curves), and $\alpha \zeta=\alpha \zeta_{\max }$ $=2000$ (dashed curves). For clarity the $\alpha \zeta=1000$ results have been omitted in the population plots. These results have been obtained by numerically solving Eqs. (13) and (14). The initial pulse was taken to be $f(\zeta=0, \tau)=\sin ^{2}\left(\pi \tau / \tau_{p}\right), \quad\left(0 \leqslant \tau \leqslant \tau_{p}\right)$, with $\tau_{p}$ being the pulse duration. The parameters used are $\tau_{p}=2000, \Gamma_{0}=10^{-3}, \delta E_{0}=$ $-5 \times 10^{-4}$, and $q=1$. For the set of figures on the left-hand side $\Delta=-1 / 2$, while for the figures on the right-hand side $\Delta=0$. The parameters are given in units of $\Gamma_{1}$. Note that the results on the left-hand side are indistinguishable.

$$
\frac{c_{0}(\zeta, \tau)}{c_{1}(\zeta, \tau)}=-\sqrt{\frac{\Gamma_{1}}{\Gamma_{0}}} \frac{1}{f^{*}(\zeta, \tau)} .
$$

Substituting this result into the equation for the pulse envelope, Eq. (14), we obtain

$$
\frac{\partial}{\partial \zeta} f(\zeta, \tau)=0
$$

with the consequence that $|f(\zeta, \tau)|^{2}=|f(0, \tau)|^{2}$ for all $\zeta$. This implies that, if the atomic medium evolves adiabatically in the laser field, the pulse will propagate at velocity $c$ and without attenuation. This is illustrated in the upper plots of Fig. 2, where we present the magnitude squared of the pulse envelope at different positions in the medium as a function of the retarded time $\tau$. We also show the corresponding populations of the bare atomic states of the system. These results have been obtained by numerically solving Eqs. (13) and (14) [33] for a sin-squared pulse and laser and atomic parameters which ensure that the system evolves adiabatically. For the set of figures on the left-hand side (lhs), the trapping conditions given by Eqs. (17) and (18) are fulfilled, whereas for the set of figures on the rhs only condition (17) is fulfilled. In the upper left-hand plot, we see that the pulse propagation is both loss- and dispersion-free, with velocity $c$ (note that the results are presented in a frame that propagates with velocity $c$ ), as given by Eq. (21). In addition, the population in the ground state returns to unity at the end of the pulse. The upper plot on the right explicitly demonstrates that when condition (18) is not satisfied the atomic populations decay and the pulse is attenuated until it is completely absorbed by the medium. We should mention that the exact fulfilment of condition (17), is not important for this particular set of parameters. This has been checked by using all the parameters of Fig. 2, except we set $\delta E_{0}=0$ such that Eq. (17) is not satisfied. The results of this calculation were in good agreement with those presented on the left-hand side of Fig. 2, i.e., transparency is established even for the case in which Eq. (17) is not exactly satisfied.

Perturbative corrections to the adiabatic behavior of the system can be calculated analytically under the assumption that the laser intensity is not too high so that $c_{0}(\zeta, \tau) \approx 1$. In this case, from Eq. (13), $c_{1}(\zeta, \tau)$ satisfies the approximate equation

$$
\left[i \frac{\partial}{\partial \tau}-\Delta+i \frac{\Gamma_{1}}{2}\right] c_{1}(\zeta, \tau)=-\frac{1}{2}(q+i) \sqrt{\Gamma_{0} \Gamma_{1}} f^{*}(\zeta, \tau) .
$$

Keeping terms up to $\partial f^{*} / \partial \tau$ in a series expansion solution of Eq. (22), we obtain

$$
\begin{aligned}
c_{1}(\zeta, \tau) \approx & \frac{(q+i) \sqrt{\Gamma_{0} \Gamma_{1}} / 2}{\Delta-i \Gamma_{1} / 2}\left[f^{*}(\zeta, \tau)\right. \\
& \left.+\frac{i}{\Delta-i \Gamma_{1} / 2} \frac{\partial}{\partial \tau} f^{*}(\zeta, \tau)\right] .
\end{aligned}
$$

Equation (23) reduces to the adiabatic solution, in the weak field limit, if the second term on the rhs is omitted. Substituting Eq. (23) into Eq. (14) we find that

$$
\left[\frac{\partial}{\partial \zeta}+\frac{1}{v} \frac{\partial}{\partial \tau}\right] f(\zeta, \tau)=i \alpha \eta f(\zeta, \tau)
$$

where we have defined the quantities

$$
\begin{gathered}
v=\frac{4\left(\Delta+i \Gamma_{1} / 2\right)^{2}}{\alpha \Gamma_{0} \Gamma_{1}(i-q)^{2}}, \\
\eta=\delta E_{0}+i \frac{\Gamma_{0}}{2}-\frac{\Gamma_{0} \Gamma_{1}(i-q)^{2}}{4\left(\Delta+i \Gamma_{1} / 2\right)} .
\end{gathered}
$$

In the case that the trapping condition Eq. (16) is satisfied, Eqs. (25) and (26) reduce to

$$
v=\frac{\Gamma_{1}}{\alpha \Gamma_{0}}, \quad \eta=0,
$$

so that

$$
\left[\frac{\partial}{\partial \zeta}+\frac{1}{v} \frac{\partial}{\partial \tau}\right] f(\zeta, \tau)=0
$$

Hence, $|f(\zeta, \tau)|^{2}=|f(0, \tau-\zeta / v)|^{2}$ for all $\zeta$, with $v$ being the group velocity of the laser pulse in the retarded frame. Therefore, as a first correction, non-adiabatic effects under population trapping conditions will modify only the group velocity of a probe laser field in the medium, in the rest frame, 


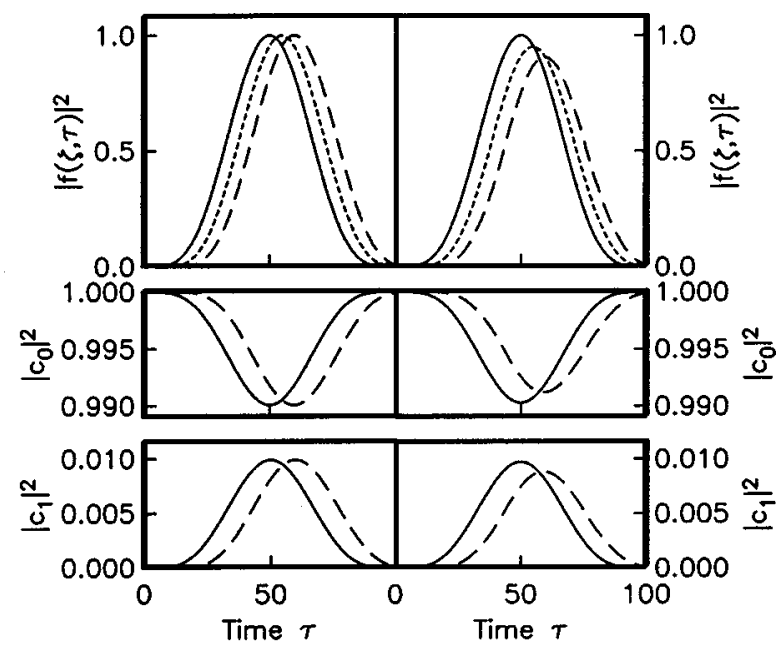

FIG. 3. Same as in Fig. 2, however, with $\tau_{p}=100, \alpha \zeta_{\max }$ $=1000, \Gamma_{0}=10^{-2}, \Delta=-5$, and $q=10$. For the figures on the lhs $\delta E_{0}=-5 \times 10^{-2}$, while on the rhs $\delta E_{0}=0$.

$$
v_{g}=c \frac{\Gamma_{1}}{\Gamma_{1}+\alpha c \Gamma_{0}}=c \frac{\Gamma_{1}}{\Gamma_{1}+2 \pi \mathcal{N} \omega \Gamma_{0} / \mathcal{E}^{2}}
$$

This is illustrated in the left-hand set of the plots of Fig. 3. These results were obtained by numerically solving Eqs. (13) and (14). It can clearly be seen that the pulse propagates without loss and that the population in the ground state returns to unity at the end of the pulse. The group velocity of the propagation determined from the numerical results is in agreement with the analytic result of Eq. (27). In the righthand set of plots, we display the results for the case that Eq. (17) is not fulfilled. This leads to absorption of the order of $15 \%$, when $\alpha \zeta=1000$. Indeed, the group velocity of the pulse is approximately the same as for the results on the left, where the trapping conditions are satisfied. We note that Eq. (28) can also be derived from Eq. (13) by taking the weakfield limit using the method discussed in Ref. [27] and obtaining the first nonadiabatic correction.

In the strongly nonadiabatic regime, ionization of the system will occur even for a weak probe pulse. Therefore, absorption and dispersion of the laser pulse will also occur, as expected, together with a change of group velocity. Despite absorption, the pulse will still propagate for many absorption lengths in the medium, when the trapping condition (16) is satisfied. We demonstrate the nonadiabatic evolution of the system in Fig. 4. It is seen that the pulse exhibits substantial absorption, of the order of $80 \%$ at $\alpha \zeta=6000$, together with dispersion. There is strong initial absorption, however, as the pulse propagates further into the medium the rate of absorption decreases since dispersion and absorption causes the interaction to become more adiabatic. This is also reflected in the behavior of the population of the ground state, which approaches unity after the pulse penetrates far into the medium. It is interesting to point out that even under these nonadiabatic conditions the group velocity of the pulse is given to good approximation by Eq. (25). Ultimately, the pulse will stabilize and propagate with only group velocity dispersion. When condition (18) is not satisfied, the pulse is almost completely absorbed at $\alpha \zeta=200$. This is shown in

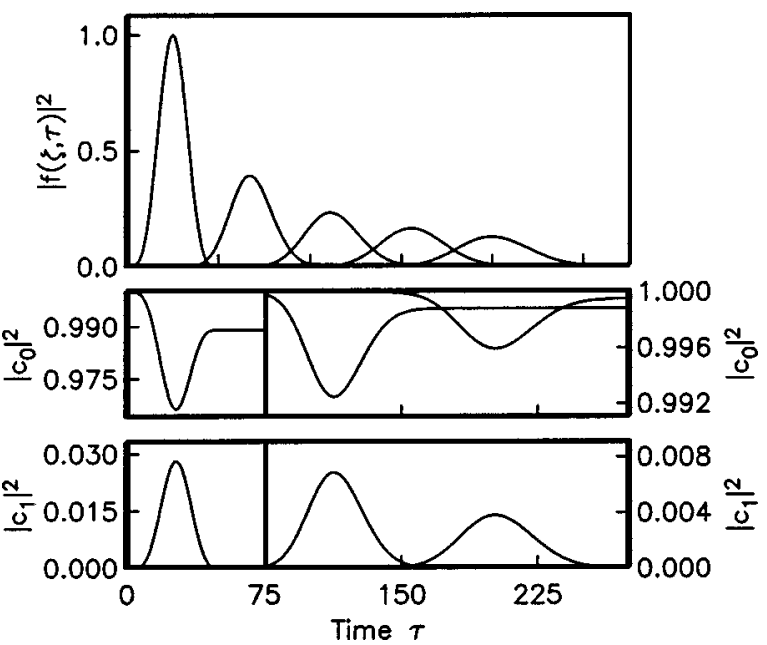

FIG. 4. Evolution of the system for a pulse duration $\tau_{p}=50$. The magnitudes squared of the pulse envelopes are shown for $\alpha \zeta=0$ to $\alpha \zeta_{\max }=6000$ in steps of $\alpha \zeta_{\max } / 4$. The populations are given in steps of $\alpha \zeta_{\max } / 2$. The parameters used are $\Gamma_{0}=3 \times 10^{-2}, \delta E_{0}=0, \Delta$ $=0$, and $q=0$.

the right-hand plot of Fig. 5. We have also checked the case when condition (17) is not satisfied by setting $\delta E_{0}=10^{-2}$. The results remain in good agreement with those obtained when $\delta E_{0}=0$, as it is seen in the left-hand plot of Fig. 5 . This illustrates once again that satisfying condition Eq. (17) does not significantly affect the transparency of the medium.

Finally, we close this section by discussing the conditions that the system must obey to establish adiabatic evolution. The adiabatic condition, under the population trapping condition Eq. (16), is given by [27]

$$
\left|\frac{\partial \theta(\zeta, \tau)}{\partial \tau}\right| \ll \frac{\sqrt{q^{2}+1}}{2}\left(\Gamma_{0}|f(\zeta, \tau)|^{2}+\Gamma_{1}\right),
$$

where $\tan \theta(\zeta, \tau)=-\sqrt{\Gamma_{0}} f^{*}(\zeta, \tau) / \sqrt{\Gamma_{1}}$. Equation (30) in the weak-field limit reads

$$
\left|\frac{\partial f(\zeta, \tau)}{\partial \tau}\right| \ll \frac{\sqrt{q^{2}+1}\left(\Gamma_{1}\right)^{3 / 2}}{2 \sqrt{\Gamma_{0}}} .
$$

For a pulse with an initial sin-squared envelope, as was used in obtaining our numerical results, the laser pulse duration, $\tau_{p}$, must satisfy

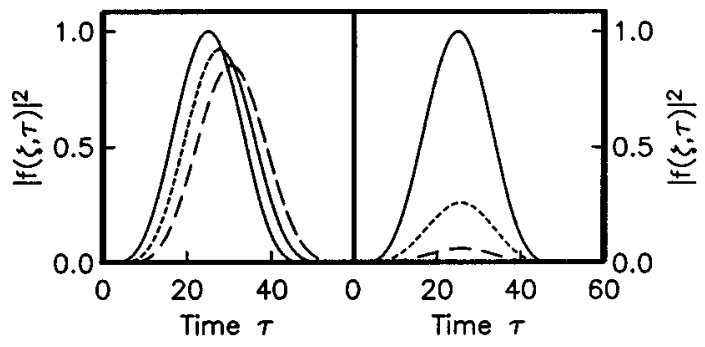

FIG. 5. Propagation in the medium of a laser pulse of duration $\tau_{p}=50$. On the lhs, results are shown for $\delta E_{0}=0$ and $\delta E_{0}=10^{-2}$ with $\Delta=0$. The results are indistinguishable. The plot on the rhs is for $\Delta=1 / 2$ and $\delta E_{0}=0$. Shown are the pulses at $\alpha \zeta$ $=0, \quad \alpha \zeta_{\max } / 2$ (dotted curves), and $\alpha \zeta_{\max }=200$ (dashed curves). The remaining parameters are the same as in Fig. 4. 


$$
\tau_{p} \gg \frac{4 \pi \sqrt{\Gamma_{0}}}{\sqrt{q^{2}+1}\left(\Gamma_{1}\right)^{3 / 2}},
$$

for adiabatic evolution to occur. This will typically correspond to picosecond laser pulses.

\section{SUMMARY AND CONCLUSIONS}

In summary, we have investigated the propagation dynamics of a short laser pulse propagating in an autoionization medium. We have demonstrated a number of effects that can occur when population trapping conditions in the system are satisfied. If the interaction of the laser field and the system is adiabatic, then the pulse will propagate intact with group velocity $c$. As a first correction to this, nonadiabatic effects lead only to a change in the group velocity of the pulse. Even in the case when nonadiabatic effects are large, the pulse remains relatively intact over a large propagation depth, under conditions of coherent population trapping. We tested these conditions with emphasis given to their effect on the propagation of a not too intense laser field in the autoionizing medium. The first condition, Eq. (17), is the most stringent, as it requires the satisfaction of a specific relation between the atomic parameters of the system. However, it was shown to have only a small effect on the transparency of the medium to the laser pulse. We close by pointing out that for an intense laser field, Eq. (17) will restrict the validity of our findings, as this condition will be as important as the one given by Eq. (18). In this case, the design of appropriately chirped laser pulses may give a possible solution to this problem [27]; however, the propagation of the space- and time-dependent phase of the pulse in the medium should also be taken into account [32].

\section{ACKNOWLEDGMENT}

This work has been supported in part by the U.K. Engineering and Physical Sciences Research Council (EPSRC).
[1] M. J. Konopnicki and J. H. Eberly, Phys. Rev. A 24, 2567 (1981).

[2] C. R. Stroud, Jr. and D. A. Cardimona, Opt. Commun. 37, 221 (1981).

[3] S. E. Harris, Phys. Rev. Lett. 70, 552 (1993); ibid. 72, 52 (1994); S. E. Harris and Z. F. Luo, Phys. Rev. A 52, R928 (1995).

[4] S. E. Harris, Opt. Lett. 19, 2018 (1994); S. E. Harris and A. V. Sokolov, Phys. Rev. A 55, R4019 (1997); Phys. Rev. Lett. 81, 2894 (1998).

[5] A. Kasapi, M. Jain, G. Y. Yin, and S. E. Harris, Phys. Rev. Lett. 74, 2447 (1995).

[6] O. Schmidt, R. Wynands, Z. Hussein, and D. Meschede, Phys. Rev. A 53, R27 (1996).

[7] L. V. Hau, S. E. Harris, Z. Dutton, and C. H. Behroozi, Nature (London) 397, 594 (1999).

[8] R. Grobe, F. T. Hioe, and J. H. Eberly, Phys. Rev. Lett. 73, 3183 (1994); F. T. Hioe and R. Grobe, ibid. 73, 2559 (1994).

[9] M. Fleischhauer and A. S. Manka, Phys. Rev. A 54, 794 (1996).

[10] I. E. Mazets, Phys. Rev. A 54, 3539 (1996).

[11] E. Cerboneschi and E. Arimondo, Phys. Rev. A 54, 5400 (1996).

[12] O. Kocharovskaya and P. Mandel, Quantum Opt. 6, 217 (1994)

[13] G. S. Agarwal, Phys. Rev. Lett. 71, 1351 (1993).

[14] M. Fleischhauer, Phys. Rev. Lett. 72, 989 (1994).

[15] G. Vemuri, G. S. Agarwal, and K. V. Vasavada, Phys. Rev. Lett. 79, 3889 (1997).

[16] J. H. Eberly, A. Rahman, and R. Grobe, Phys. Rev. Lett. 76, 3687 (1996).

[17] G. Vemuri, K. V. Vasavada, G. S. Agarwal, and Q. Zhang, Phys. Rev. A 54, 3394 (1996).

[18] A. Rahman and J. H. Eberly, Phys. Rev. A 58, R805 (1998).

[19] B. M. Garraway and K. A. Suominen, Phys. Rev. Lett. 80, 932 (1998).
[20] J. R. Csesznegi and R. Grobe, Phys. Rev. Lett. 79, 3162 (1997); J. R. Csesznegi, B. K. Clark, and R. Grobe, Phys. Rev. A 57, 4860 (1998).

[21] S. J. van Enk, J. Zhang, and P. Lambropoulos, Phys. Rev. A 50, 2777 (1994).

[22] F. T. Hioe and C. E. Carroll, Phys. Rev. A 56, 2292 (1997).

[23] U. Fano, Phys. Rev. 124, 1866 (1961).

[24] E. Paspalakis, N. J. Kylstra, and P. L. Knight, Phys. Rev. Lett. 82, 2079 (1999).

[25] B. W. Shore, Phys. Rev. 171, 43 (1968); J. P. Connerade, Highly Excited Atoms (Cambridge University Press, Cambridge, 1997), Chap. 6, and references therein.

[26] J. Zhang, S. J. van Enk, and P. Lambropoulos, Opt. Commun. 125, 168 (1996).

[27] E. Paspalakis, M. Protopapas, and P. L. Knight, J. Phys. B 31, 775 (1998).

[28] For a recent review on adiabatic processes in laser fields, see K. Bergmann, H. Theuer, and B. W. Shore, Rev. Mod. Phys. 70, 1003 (1998).

[29] For a recent review on coherent population trapping, see E. Arimondo, in Progress in Optics XXXV, edited by E. Wolf (Elsevier, Amsterdam, 1996), p. 257.

[30] P. Lambropoulos and P. Zoller, Phys. Rev. A 24, 379 (1981).

[31] K. Rzążewski and J. H. Eberly, Phys. Rev. Lett. 47, 408 (1981).

[32] L. Allen and J. H. Eberly, Optical Resonance and Two-Level Atoms (Dover, New York, 1987), Chaps. 1, 4, and 5.

[33] B. W. Shore, The Theory of Coherent Atomic Excitation Vol. 1 (Wiley Interscience, New York, 1989), Chap. 12.

[34] J. H. Eberly, M. L. Pons, and H. R. Haq, Phys. Rev. Lett. 72, 56 (1994); J. H. Eberly, Quantum Opt. 7, 373 (1995).

[35] See, for example, N. J. Kylstra, E. Paspalakis, and P. L. Knight, J. Phys. B 31, L719 (1998).

[36] O. Latinne, N. J. Kylstra, M. Dörr, J. Purvis, M. TeraoDunseath, C. J. Joachain, P. G. Burke, and C. J. Noble, Phys. Rev. Lett. 74, 46 (1995). 